\title{
Genetic mapping of legume orthologs reveals high conservation of synteny between lentil species and the sequenced genomes of Medicago and chickpea
}

\author{
Neha Gujaria-Verma ${ }^{1}$, Sally L. Vail ${ }^{1,2+}$, Noelia Carrasquilla-Garcia ${ }^{2}$, R. Varma Penmetsa ${ }^{2}$, \\ Douglas R. Cook ${ }^{2}$, Andrew D. Farmer ${ }^{3}$, Albert Vandenberg ${ }^{1}$ and Kirstin E. Bett ${ }^{1 *}$
}

'Department of Plant Sciences, University of Saskatchewan, Saskatoon, SK, Canada
2 Department of Plant Pathology, University of California, Davis, Davis, CA, USA
${ }^{3}$ Bioinformatics, National Centre for Genomic Resources, Santa Fe, NM, USA

Edited by:

Paul Gepts, University of California,

Davis, USA

Reviewed by:

Eduard Akhunov, Kansas State

University, USA

Marc Libault, University of

Oklahoma, USA

*Correspondence:

Kirstin E. Bett, Pulse Crop Breeding and Genetics, Department of Plant

Sciences, University of

Saskatchewan, 51 Campus

Dr., Saskatoon, SK, Canada

e-mail:k.bett@usask.ca

${ }^{\dagger}$ Present address:

Sally L. Vail, Agriculture and

Agri-Food Canada, Saskatoon

Research Centre, Saskatoon, SK,

Canada
Lentil (Lens culinaris Medik.) is a global food crop with increasing importance for food security in south Asia and other regions. Lens ervoides, a wild relative of cultivated lentil, is an important source of agronomic trait variation. Lens is a member of the galegoid clade of the Papilionoideae family, which includes other important dietary legumes such as chickpea (Cicer arietinum) and pea (Pisum sativum), and the sequenced model legume Medicago truncatula. Understanding the genetic structure of Lens spp. in relation to more fully sequenced legumes would allow leveraging of genomic resources. A set of 1107 TOGbased amplicons were identified in L. ervoides and a subset thereof used to design SNP markers for mapping. A map of L. ervoides consisting of 377 SNP markers spread across seven linkage groups was developed using a GoldenGate genotyping array and single SNP marker assays. Comparison with maps of $M$. truncatula and $L$. culinaris documented considerable shared synteny and led to the identification of a few major translocations and a major inversion that distinguish Lens from $M$. truncatula, as well as a translocation that distinguishes $L$. culinaris from $L$. ervoides. The identification of chromosome-level differences among Lens spp. will aid in the understanding of introgression of genes from $L$. ervoides into cultivated $L$. culinaris, furthering genetic research and breeding applications in lentil.

Keywords: wild lentil, legume tentative orthologs, mapping, translocation, synteny, Medicago

\section{INTRODUCTION}

Lentil (Lens culinaris Medik.; Lc) is the fifth most important grain legume crop globally and an important export crop for Canada. It is cultivated primarily in North America, Australia, the Middle East, Western Asia, and the Indian subcontinent (Erskine, 1996). It is self-pollinating with a diploid genome $(2 \mathrm{n}=14)$ of $4 \mathrm{Gbp}$ (Arumuganathan and Earle, 1991). Similar to other legume crops, lentil fixes atmospheric nitrogen and provides rotational and environmental benefits, such as management of insect pests, diseases, and weeds. It is a valuable cash crop and provides an important source of dietary protein, carbohydrates, and vitamins for the human diet. The small seeds cook quickly, giving this crop a culinary advantage over other grain legumes.

Narrow genetic diversity limits genetic improvement of lentil, while susceptibility to diseases and weed infestations are the main production constraints. Wild relatives are valuable sources of resistance genes for crop species, and introgression of these genes plays an important role in increasing genetic variation (Zhao et al., 2010; Cornille et al., 2012; Hufford et al., 2013). The secondary gene pool of cultivated lentil, especially L. ervoides (Le), is a source of resistance to anthracnose races Ct0 and
Ct1 (Tullu et al., 2006; Fiala et al., 2009; Vail et al., 2011), stemphylium blight (Podder et al., 2013), and ascochyta blight (Bayaa et al., 1994). Inter-specific hybrids derived from a cross between Lc and Le show significant resistance to anthracnose, likely derived from genes present in the wild parent (Tullu et al., 2006).

The development of molecular markers has accelerated trait mapping studies in legumes (Gupta and Varshney, 2000; Andersen and Lübberstedt, 2003; Cannon et al., 2009; Varshney et al., 2009), and gene-based molecular markers are emerging as important tools for molecular mapping in lentil (Hamwieh et al., 2005; Alo et al., 2011; Kaur et al., 2011; Fedoruk et al., 2013). Single-nucleotide polymorphism (SNP)-based markers are ideal for diversity assessment and genetic mapping in crop species due to their high abundance, relatively even distribution across the genome, and the virtual absence of homeoplasy (Varshney, 2010; Gujaria et al., 2011; Sharpe et al., 2013). Lentil has lagged in genomic resource development because of its minor status relative to large global grain crops and its large genome size, though recent efforts by several groups have led to an increase in availability of sequence datasets (Kaur et al., 2011; Sharpe et al., 2013). Rapid advances in massively parallel sequencing and genotyping 
have revolutionized genomics by reducing both its cost and time by several orders of magnitude (Mardis, 2008; Metzker, 2009; Varshney, 2010). Among the high-throughput genotyping platforms available, the GoldenGate assay from Illumina Inc. (San Diego, CA) has proven to be efficient and useful for genetic fingerprinting (Hyten et al., 2010; Yan et al., 2010; Zhao et al., 2010; Blair et al., 2013). GoldenGate assays have been developed for several legume crops, including common bean (Blair et al., 2013), soybean (Hyten et al., 2008), cowpea (Muchero et al., 2009), pea (Deulvot et al., 2010), chickpea (Gaur et al., 2012), and most recently, lentil (Sharpe et al., 2013).

Lentil belongs to the galegoid sub-family of the Papilionoideae and is a member of the Vicieae tribe. Vicieae consists of the important crop and model legumes Medicago truncatula (Mt), lentil, field pea (Pisum sativum L.) and faba bean (Vicia faba L.). Most of the genes in papilionoid legume species are likely to be found within syntenic regions ranging in size from hundreds of kilobases to several megabases with respect to any other given papilionoid species (Cannon et al., 2009). If a gene and phenotype are experimentally associated in one species, then an orthologous gene is likely to be found in a similar genomic region in closely related species. Taking advantage of this makes comparative genetic mapping a powerful tool for establishing shared ancestry between species and leveraging information from more well-studied species. Genomic relationships have been charted between pea and lentil (Weeden et al., 1992), Mt and pea (Choi et al., 2004a,b; Kalo et al., 2004), and Lc and Mt (Phan et al., 2006; Alo et al., 2011; Sharpe et al., 2013).

Conserved orthologous sequences (COS) have been used to derive markers from a set of low-copy orthologous genes (tentative orthologous genes; TOGs) present in a legume expressed sequence tag (EST) dataset of Mt, lotus (Lotus japonica) and soybean (Glycine max) transcripts (Penmetsa et al., unpublished). The fact that TOGs have retained their mostly single-copy nature during speciation, they can be helpful in the study of genome structure and evolution across species (Fulton et al., 2002; Choi et al., 2006). Legume TOGs have been exploited to trace the evolutionary history of pigeonpea (Cajanus cajan) (Kassa et al., 2012) and common bean (Phaseolus vulgaris) (Blair et al., 2013). Mapping of these low copy number sequences in chickpea (Hiremath et al., 2012) and pigeonpea (Saxena et al., 2012) has also helped in the comparison of their genome structures with those of Mt, lotus and soybean (Cook et al., unpublished). Conserved synteny among legume species is often punctuated by chromosomal rearrangements, reflected by differences in chromosome numbers among their genomes. These chromosome rearrangements can be explained by translocations or inversions within legume species (Choi et al., 2004a). Ladizinsky et al. (1984) concluded that L. nigricans and L. lamottei differ by four reciprocal translocations and one paracentric inversion, resulting in the complete sterility of hybrids with Lc. Ladizinsky et al. (1985) and Tadmor et al. (1987) used crosses between Lc and Le to conclude that a single reciprocal translocation differentiates the two species. Weeden et al. (1992) used one of those interspecific populations and isozyme markers to map the translocation region.

This study was undertaken to capitalize on the recent development of genomic resources in lentil, with objectives to: (i) sequence intron spanning amplicons from the legume TOGs in lentil, (ii) generate TOG-based SNP genotyping assays, (iii) develop a SNP-based genetic map of L. ervoides, and (iv) determine macrosyntenic relationships between wild and cultivated lentil and with those of closely related galegoid legumes.

\section{MATERIALS AND METHODS DNA AMPLIFICATION AND SEQUENCING}

Le accessions IG 72815 (from Turkey) and L01-827a (a single plant selection from IG 72847; Fiala et al., 2009), and Lc cultivars "CDC Redberry" (Vandenberg et al., 2006) and "Eston" (Slinkard, 1981) were selected for TOG marker development. DNA was extracted from freeze-dried leaf tissue of 2-week-old seedlings by a modified CTAB method (Doyle and Doyle, 1990). The quality and quantity of DNA were assessed using $1 \%$ agarose gel and a FLUOstar Omega flourimeter (BMG Labtech). DNA was normalized to $50 \mathrm{ng} / \mu \mathrm{L}$ for sequencing and genotyping.

LR-66 is a population developed from a cross between the two Le accessions. An $\mathrm{F}_{2}$ population of 91 individuals was generated. LR-59 is an inter-species cross derived from a cross between Eston (Lc) and L01-827a (Le). A F F:8 RIL population of 68 individuals had already been developed (Fiala et al., 2009). DNA for LR-66 and LR-59 was extracted and quantified as described above for the Le accessions and Lc cultivars.

\section{SANGER SEQUENCING AND SNP IDENTIFICATION}

PCR amplicons for Sanger sequencing were generated using primers designed to amplify 1440 TOGs (Penmetsa et al., unpublished) using Le genotypes IG 72815 and L01-827a, and Lc genotypes CDC Redberry and Eston as DNA templates. Sequencing from both $3^{\prime}$ - and $5^{\prime}$-ends using forward and reverse primers was carried out and the resulting forward and reverse read amplicon sequences were first assembled for each genotype using CAP3 (Huang and Madan, 1999). The resulting contigs along with any unassembled reads were included in another round of assembly by CAP3 to produce a master alignment from which SNPs were identified using an in-house bioinformatics pipeline at the National Center for Genome Resources (NCGR; Santa Fe, NM). All SNPs were manually curated to verify the alleles called by the software. All contigs and identified SNPs are available through the KnowPulse webportal at http://knowpulse2.usask.ca/portal/ node/53 and in Additional file $1 \mathrm{~b}$.

\section{SNP GENOTYPING \\ IIlumina GoldenGate assay}

To design an Illumina GoldenGate Oligonucleotide Pool Assay (OPA), a set of 9575 validated SNPs was submitted to Illumina for Assay Design Tool (ADT) scoring. Only SNPs with an ADT score of 0.5 or higher were considered for OPA design. SNPs were examined for informativeness based on anticipated polymorphism between Lc and Le or Le and Le mapping populations, and to maximize the number of TOGs covered. A set of 768 SNPs were selected from this filtering and sent to Illumina for the synthesis of the Le768 SNP OPA assay.

The $91 \mathrm{~F}_{2}$ individuals from the LR-66 population and 68 LR-59 RILs were genotyped using this Le768 OPA and the standard Illumina GoldenGate Assay protocols 
(http://www.illumina.com/technology/goldengate_genotyping_ assay.ilmn). The products generated by this assay were read with an Illumina iScan (Illumina Inc., San Diego, CA). The resulting data were clustered for allele calling using GenomeStudio software version 2010.3 (Illumina, San Diego, CA). Allele calls were manually checked for errors and corrected as necessary or scored as missing data to exclude their further use. To avoid additional technical errors, all SNPs showing unexpected parental alleles or high levels of missing data $(>10 \%)$ were excluded from further analysis.

\section{Single SNP assays}

An additional 31 gene-based SNPs, polymorphic between L01-827A and IG 72815, were identified through a separate project (Sharpe et al., 2013). Allele-specific forward primers and a common reverse primer were designed for use in KASP ${ }^{\text {тм }}$ (Kompetitive Allele Specific PCR) assays (LGC Genomics Ltd. Hoddesdon, U.K.; http://www.lgcgenomics.com/ kasp-genotyping-reagents). Assays were carried out in a $10 \mu \mathrm{L}$ reaction volume consisting of $50 \mathrm{ng} / \mu \mathrm{L}$ DNA, KASP $1 X$ Reaction Mix, and $0.17 \mu \mathrm{M}$ KASP Assay Mix (allele-specific primers and common primer). PCR amplification was carried out in StepOnePlus ${ }^{\mathrm{TM}}$ Real-Time PCR System (Applied Biosystems) and fluorescence was analyzed using StepOne Software version 2.1 (Applied Biosystems). Allele calling was done graphically as scatter plots for each marker using SNPViewer (LGC Genomics Ltd., Hoddesdon, UK).

A total of 110 TOG-based SNPs were identified between the two Lc lines for which there was sufficient flanking sequence to design allele-specific primers for KASP assays. Sequence information was sent to KBioscience (now LGC Genomics Ltd.) for assay design and genotyping on the parents of mapping populations, including LR-66.

\section{GENETIC MAPPING}

LR-66 (Le $\times$ Le) $\mathrm{F}_{2}$ individuals were genotyped using the Le768 OPA and KASP assays. Polymorphic loci were used for mapping using the regression mapping function in JoinMap 4.0 (Van Ooijen, 2006) with a minimum LOD value of 5.0. A Kosambi mapping function was used to convert recombination frequency into centimorgan (cM) distances. Linkage maps were drawn with MapChart version 2.1 (Voorrips, 2002).

\section{COMPARATIVE MAPPING WITH CLOSELY RELATED LEGUMES}

A comprehensive genetic map of Lc based on the mapping population LR-18 (Lc $\times$ Lc) developed by Fedoruk et al. (2013) was compared with the Le map (LR-66) developed in this study. Due to a lack of common markers, it was necessary to align both to the Medicago genome (version 3.5.1) to facilitate the comparison. Homologs between Le and Mt as well as Lc and Mt were identified using the NUCmer pipeline of the MUMmer software (Kurtz et al., 2004). The visual comparison of these two maps with $\mathrm{Mt}$ was made using Strudel, a freely available software package (Bayer et al., 2011; http://bioinf.hutton.ac.uk/strudel/).

Genotypic data for the interspecies RIL population, LR-59 (Le $\times$ Lc; data not shown), were used to further examine putative translocations. Common markers for Le linkage groups (LG)
LeLG1, LeLG4, LeLG5, and LeLG7 were selected and their order across individuals the Le $\times$ Lc inter-specific population LR-59 examined visually.

The orthologous map positions of TOGs mapped in Le were extracted from the Legume Information System (LIS; http:// cmap.comparative-legumes.org) for chickpea and Mt. Map units for lentil and chickpea were converted to base pair distances to facilitate comparisons with $\mathrm{Mt}$ at an assumed factor of 250,000 bp per cM. Inter-genomic comparisons were drawn using Circos, which is a Perl script based visualization tool to facilitate the identification and analysis of synteny and differences between the genomes arising from comparative analysis (Krzywinski et al., 2009; http://circos.ca/). For a better comparison of the translocation on Lc (Sharpe et al., 2013) with respect to Le, dotplots were generated for Le/Mt as well as Le/Ca by aligning the TOG member sequences to the genome using GMAP (Wu and Watanabe, 2005), then visualizing the correspondences between the physical positions and the genetically derived locations using the CMTV software (Sawkins et al., 2004).

\section{RESULTS}

\section{TOG SEOUENCING AND SNP DISCOVERY}

Sequence data were obtained for 1107 of 1440 TOG primer sets used, across all four genotypes. A total of 9575 polymorphisms were identified in 1100 unique genes (Additional File 1a). Of these, 2513 were reported as indels or multiple nucleotide polymorphisms (MNPs). The remaining 7062 SNPs were found to be polymorphic in either of the four genotypes and could be present in any of the combinations derived from these genotype $($ Lc $\times$ Lc; Lc $\times$ Le; Le $\times$ Le; Figure 1A). The largest numbers of SNPs $(6723 ; 9570.2 \%)$ were identified between Le and Lc, with fewer $(2425 ; 34.3 \%)$ between the two Le wild accessions, and only $625(8.8 \%)$ between the two Lc accessions. Although only 45 SNPs $(0.63 \%)$ were common to all three comparisons (Lc vs. Le, Le vs. Le, and Lc vs. Lc), many more SNPs (2088; 29.6\%) were common to both Le vs. Le and Lc vs. Le combinations (Figure 1A).

\section{SNP ASSAY DEVELOPMENT AND GENOTYPING}

Of the 9575 SNPs submitted to Illumina for Assay Design Tool (ADT) scoring, only 1470 scored higher than 0.5 and were considered for possible inclusion in the OPA design. The 1470 SNPs represented polymorphism in 765 non-redundant genes. For each gene, a single SNP, maximally polymorphic among the four genotypes, was chosen for inclusion in a 768-SNP GoldenGate assay. The majority of SNPs $(81.8 \%)$ were polymorphic between at least one Lc and at least one Le genotype, $61.6 \%$ of the SNPs were polymorphic between the two Le genotypes, and $16.7 \%$ were polymorphic between the two Lc genotypes (Figure 1B). The most informative markers were a set of 19 SNPs that were present between the two Lc, the two Le, and at least one Lc and one Le genotype. An additional three SNPs identified in the 765 genes were included to constitute a 768-SNP GoldenGate array for Le (Additional File 1b).

Of the 768 SNPs genotyped via the GoldenGate assay on the LR-66 population, 66 (8.6\%) failed and an additional 61 (7.9\%) were difficult to score (Additional File 1b). Only 29 marker loci 


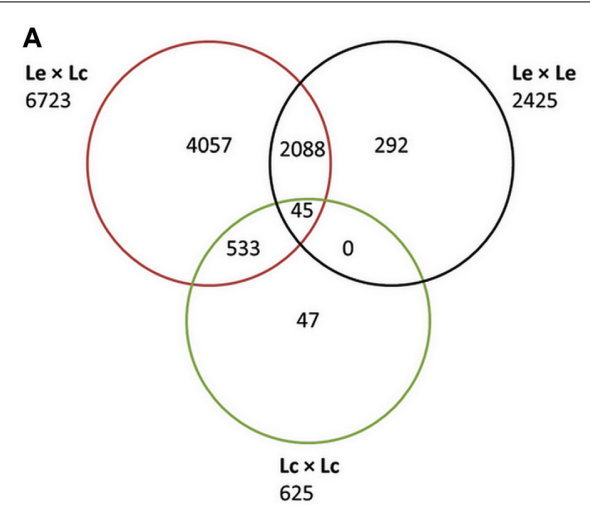

FIGURE 1 | Venn diagram partitioning out the informativeness of total SNPs between Lens culinaris (Lc), between L. ervoides (Le), and between the two species (Le and Lc) and their intersections. (A)

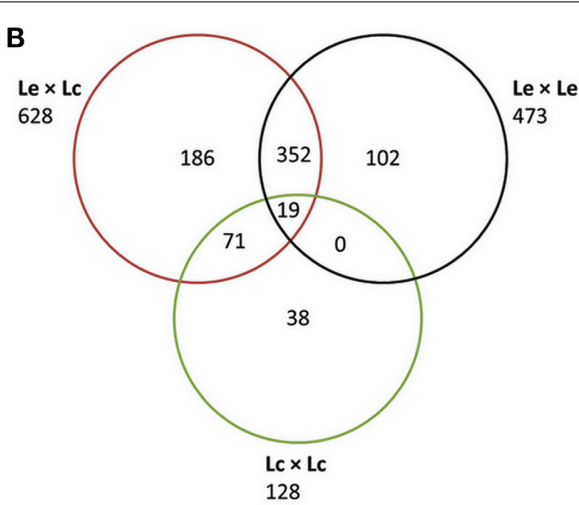

Classification based on all SNPs discovered using Sanger sequencing of CDC Redberry (Lc), Eston (Lc), L01-827a (Le), and IG 72815 (Le). (B) Classification of the subset of SNPs selected for the Le768 Illumina GoldenGate OPA.
$(8 \%)$ were severely distorted $(p<0.01)$ and all were skewed toward the Le parent, IG 72815. Of the 31 SNPs from Sharpe et al. (2013) that were genotyped via KASP assays, 20 were polymorphic in the LR-66 population (Additional File 1c). Based on Sanger sequencing data for the Le parents of LR-66, 392 loci were expected to be polymorphic and segregating in the $\mathrm{F}_{2}$ population; indeed, 363 loci yielded data amenable for use in linkage mapping (Additional File 1b).

Of the 110 KASP assays designed to monitor SNPs in TOGs, 10 failed to amplify fragments. Of the 100 remaining, 71 were polymorphic when tested on the two Lc genotypes, CDC Redberry and Eston but only 11 of the 71 were segregating in the Lc mapping population (LR-18: CDC Robin $\times 964 a-46)$ and could be mapped along with additional SNPs (Fedoruk et al., 2013). None of these 11 markers were polymorphic in the Le population LR-66.

\section{FIRST GENERATION GENETIC MAP OF L. ERVOIDES}

A total of 377 markers mapped into seven linkage groups (LeLG1LeLG7; Figure 2), which likely represent the seven chromosomes of Le. This genetic map spans $973.7 \mathrm{cM}$ with an average intermarker distance of $2.6 \mathrm{cM}$. Similar numbers of marker loci mapped on to each linkage group, varying from 50 (LeLG1 and LeLG7) to 62 (LeLG4). The linkage groups were numbered to best match those in the Lc map of Sharpe et al. (2013).

\section{COMPARATIVE MAPPING WITH CLOSELY RELATED LEGUMES}

Of the TOGs that mapped on the seven linkage groups of Le, 315 (99\%) had orthologs located on the eight chromosomes of $\mathrm{Mt}$ and 128 of these had orthologs that also mapped to the eight chromosomes of chickpea (Additional file 2). When the maps of the three species were aligned, extensive stretches of shared collinearity among them were evident (Figure 3; Additional file 3). A minimum of 41 (LeLG1) and a maximum of 56 (LeLG4) TOGs per Le linkage group had corresponding loci mapped in Mt (Additional file 2). One of the TOGs (TOG963130) was on a Mt scaffold that had not yet been incorporated into a Mt chromosome but mapped on Le-LG4 at $44.5 \mathrm{cM}$. A closer look at the comparison between Le and $\mathrm{Mt}$ revealed that Mt Chromosome 6 is collinear with the middle of LeLG2 (Figure 3B). There is a major translocation involving Mt chromosomes 4 and 8 relative to LeLG4 and LeLG7, and major inversions within LeLG1 relative to Mt chromosome 1 and within LeLG3 relative to Mt chromosome 3 were also evident.

The 128 TOGs mapped in Ca, Mt and Le, covered all Ca chromosomes with 8-22 TOGs per chromosome and corresponded to loci on all 7 Le linkage groups with 9-21 TOGs per linkage group (Additional file 2). As was seen with the Le-Mt comparison, there were large stretches of conserved synteny between Le and $\mathrm{Ca}$ (Additional file 3). The additional chromosome in $\mathrm{Ca}$ appears to result from a splitting into chromosomes 5 and 6 of the ancestral chromosome that corresponds to LeLG5 into Ca.

\section{IDENTIFYING TRANSLOCATIONS IN L. ERVOIDES vS. L. CULINARIS}

A high degree of collinearity was observed for the two lentil species in comparison with $\mathrm{Mt}$, but a few key differences were also apparent (Figure 4A). Most notably, the break in conserved synteny observed on LcLG1 and LcLG5 with respect to Mt chromosomes 1 and 5 (Sharpe et al., 2013) was not observed in Le (Figure 4B). A translocation that occurs in Le with segments of LeLG4 and LeLG7 with respect to Mt chromosomes 4 and 8 is not as apparent in the LR-18 Lc linkage map (Sharpe et al., 2013; Figure 4C). In a different Lc mapping population (LR-03) derived from the cross ILL $1704 \times$ ILL 7537, however, additional markers mapped on LcLG4 and LcLG7 (unpublished data). These are monomorphic in LR-18 but homologous to Mt chromosomes 8 and 4 , respectively, suggesting the same translocation with respect to Mt occurs in both Lens species.

Genotypic data from one of the linkage groups from the Lc $\times$ Le interspecies introgression population, LR-59, were significantly distorted (69\% skewed) toward the Lc parent alleles and aligned with portions of both LeLG1 and LeLG5 (Figure 5). Disproportionate numbers of heterozygous loci were evident in the region surrounding the break in collinearity in this linkage group. Segregation distortion or breaks in collinearity were not observed in the LR-59 linkage groups that correspond to LeLG4 and LeLG7 (data not shown). 

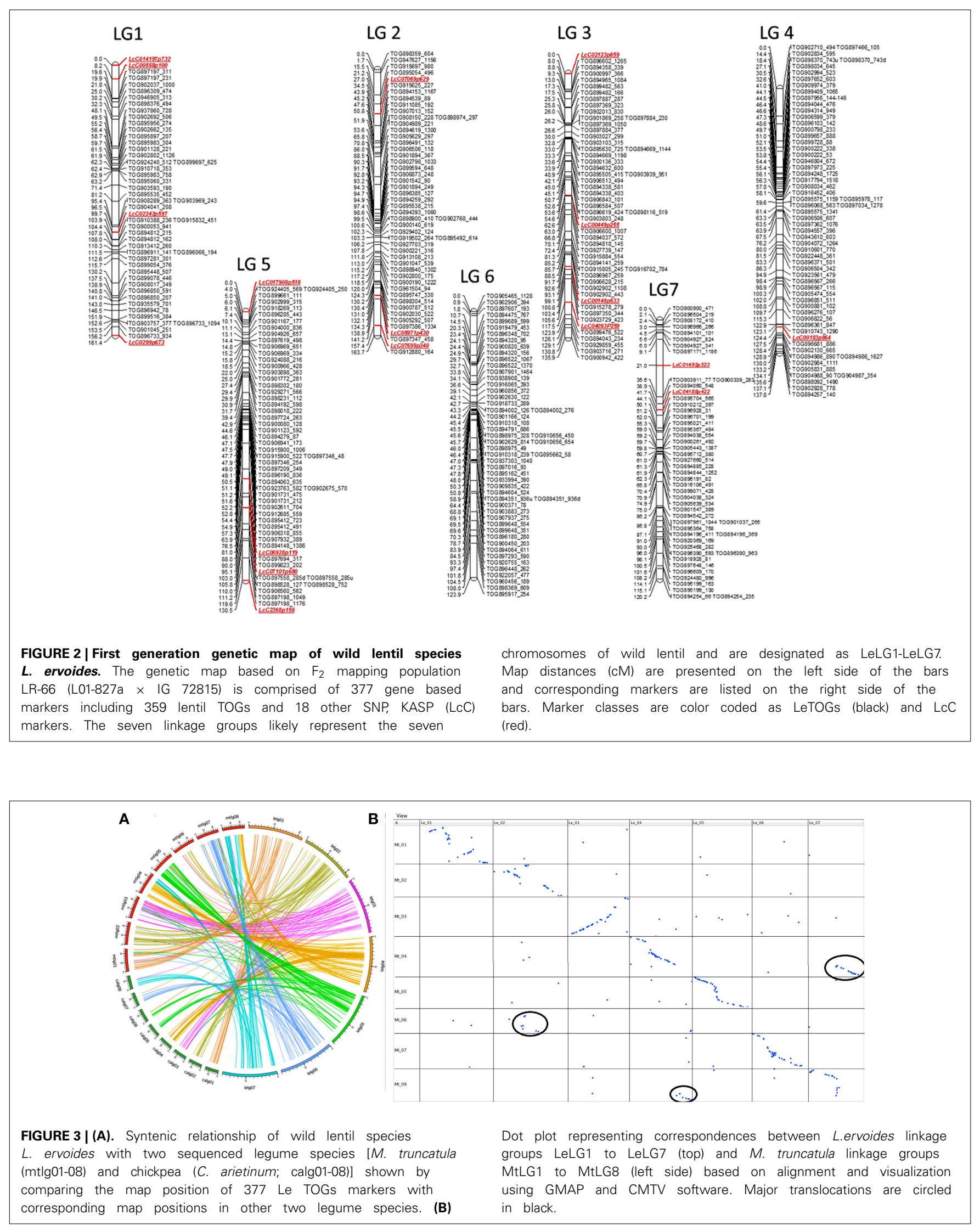

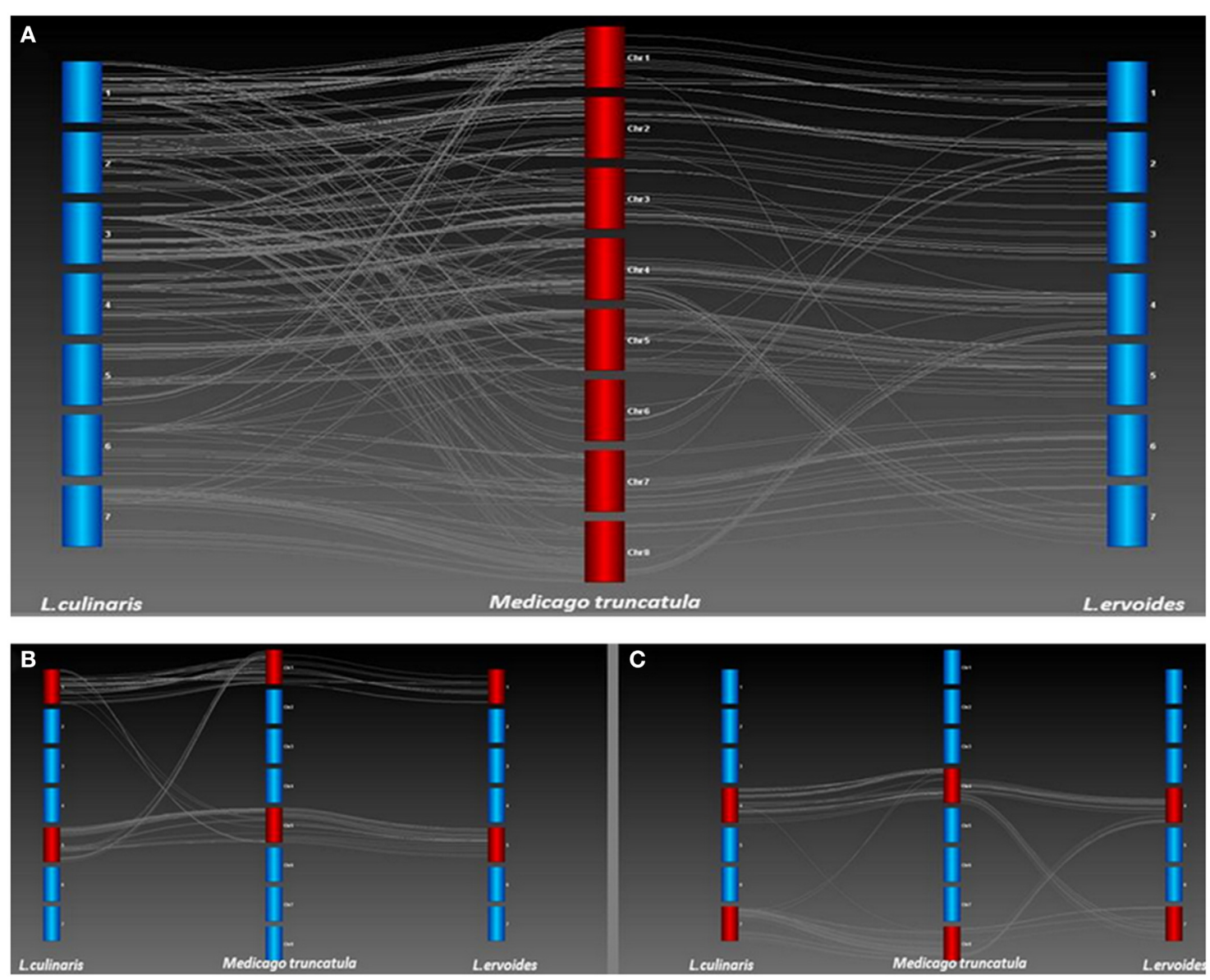

FIGURE 4 | (A) Collinearity between L. culinaris (LC) and L. ervoides (Le) with respect to $M$. truncatula (Mt). Genetic maps of Lc and Le were compared with the Mt 3.5.1 genome build (Lavin et al., 2005) to identify chromosomal re-arrangements. Each bar represents a linkage group of Lc or Le or chromosome of Mt. Lines between linkage groups and Mt chromosomes represent Homologs as determined through a BLAST of the Lens sequences against the Mt genome. (B) A translocation was identified on LG1 and LG5 of Lc with respect to Mt chromosomes 1 and 5 but not observed in Le LR-66. (C) A translocation was identified on LG4 and LG7 of Le with respect to chromosomes 4 and 8 of Mt but not observed in Lc LR-18.

\section{DISCUSSION \\ SNP DISCOVERY AND GENOTYPING}

Until recently, microsatellites and amplified fragment length polymorphisms (AFLP) were the marker systems of choice for lentil molecular marker based linkage studies. With the advent of high-throughput sequence technology, thousands of SNPs have now been identified in lentil germplasm (Kaur et al., 2011, 2013; Sharpe et al., 2013) and new, SNP-based molecular markers have been developed (Kaur et al., 2013; Sharpe et al., 2013). These high-throughput SNP genotyping platforms are useful for rapidly genotyping mapping populations and germplasm. Several SNP genotyping methods have been developed, such as array methodologies (Sapolsky et al., 1999; Ishikawa et al., 2005), pyrosequencing (Ronaghi et al., 1998; Ahmadian et al., 2000; Oliphant et al., 2002), and other high throughput techniques. Illumina GoldenGate assays have been used extensively because of their high throughput nature, multiplexing capability, accuracy, speed, and low cost per data point compared to other genotyping platforms. This genotyping platform is suitable for large-scale
SNP genotyping but is not economical for small numbers of SNPs. KBioscience (now LCG Genomics) developed KASP assays to target SNPs and indels. These assays are simple and costeffective, and are well adapted to low- to medium-throughput genotyping projects (Chen et al., 2010).

SNPs identified in this study originated from Sanger sequencing of a set of legume TOGs in two Le (wild lentil) and two Lc (cultivated lentil) accessions. These SNPs were either polymorphic in cultivated lentil (Lc-Eston vs. Lc-Reberry), wild lentil (Le-L01-827a vs. Le-IG72815) or cultivated vs. wild (Lc vs. Le; Eston/L01-827a, Eston/IG72815, Redberry/L01-827a, Redberry/IG72815). As expected, due to the occurrence of the same SNP in multiple groups, the total number of the SNPs from each group has exceeded the final SNPs (9575; Figure 1A). The majority of the SNPs were between Le and Lc, in part because there were twice as many possible comparisons but also because these are divergent species. Many more SNPs were observed between the two wild Le accessions and far fewer were observed between the two cultivated Lc accessions. Although the two Lc 


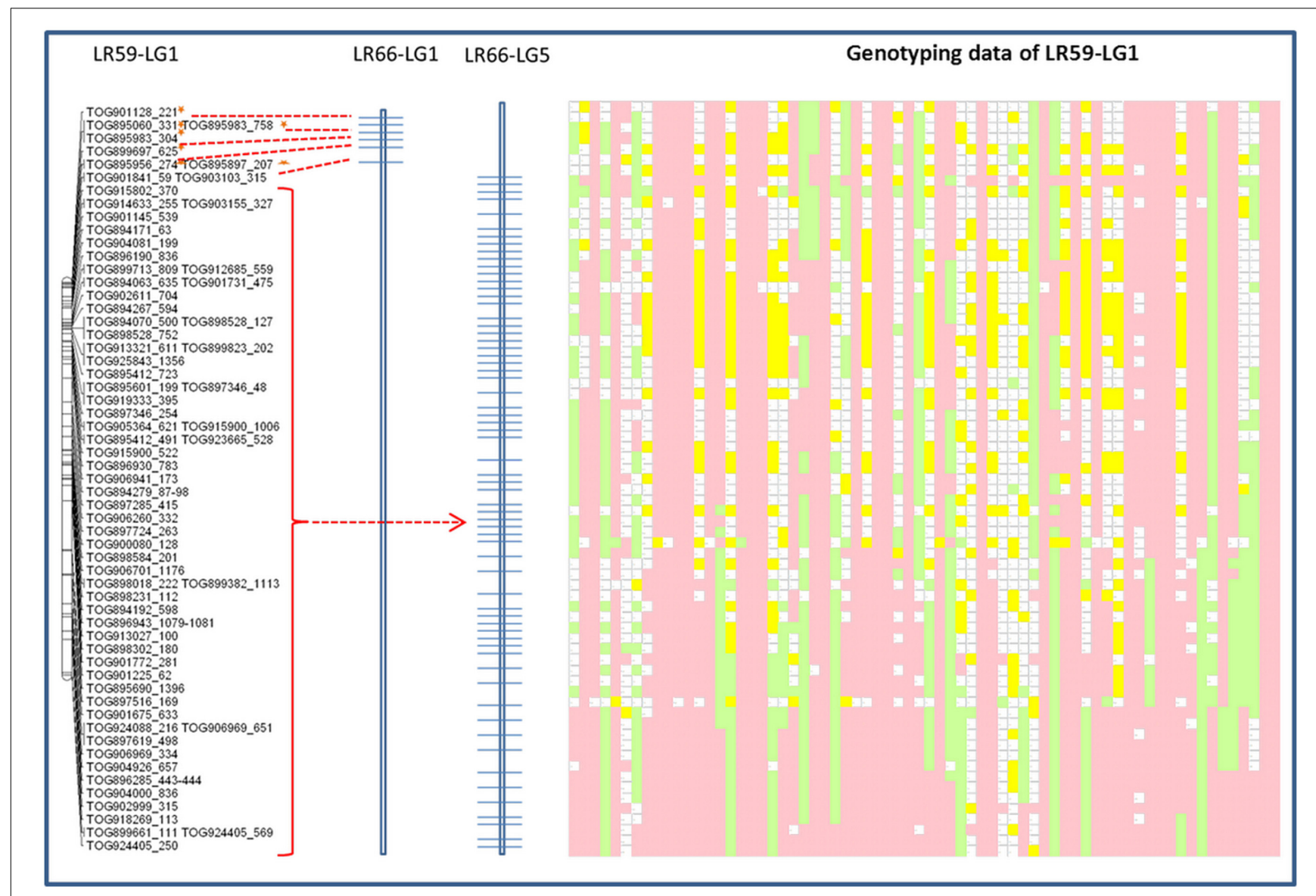

FIGURE 5 | Schematic representation of the distribution of Le768 OPA assay TOGs mapped in LG1 in the interspecific LR-59 population.

Common markers mapped in the LR-66 population are represented on

LR-66-LG1 and LR-66-LG5. Segregation of the same common markers in
LR-59 is depicted by genotyping data. Each column represents an individual RIL and each row represents a locus genotyped using the TOGs. Pink blocks represent Eston (LC) alleles, green blocks represent L01-827a (Le) alleles, yellow blocks are heterozygous loci, and blank blocks represent missing data. accessions represent the two major commercial classes of lentils (small red and small green) grown in Canada, they are both adapted to western Canadian growing conditions and likely do not represent the maximum genetic diversity that exists in the Lc genepool.

Due to the occurrence of more polymorphisms in Le and between the wild and cultivated species, an Illumina GoldenGate OPA was more practical for linkage mapping in wild lentil and interspecies hybrid populations, but not for linkage mapping within cultivated Lc. The Le768 OPA was used successfully to genotype an $\mathrm{F}_{2}$ mapping population, LR-66, derived from a Le $\times$ Le cross. Only $8.6 \%$ of the loci failed, which is consistent with other experiences with unvalidated OPAs (Rostoks et al., 2006; Hyten et al., 2008). The OPA was not used to genotype the Lc $\times$ Lc mapping population as the majority of the loci were expected to be monomorphic.

In LR-66, a Le $\times \mathrm{Le} \mathrm{F}_{2}$ population, 392 loci were anticipated to be polymorphic based on the Sanger sequencing results of parental lines, and we obtained high quality genotypic data for 363 loci. The reduced number of segregating loci were explained by parental genotypes at 29 loci $(8.0 \%)$ that did not match with the Sanger sequence genotypes for the two accessions and a further 20 loci genotyped as heterozygous in one or other of the mapping parents but not reported as such in the Sanger data. Heterogeneity of parental seed stocks or residual heterozygosity in plants used for DNA sequencing vs. those used to develop the LR66 population may underlie such genotypic differences. Another problem encountered with 61 loci, caused by trying to map an $\mathrm{F}_{2}$ population, was the difficulty in distinguishing heterozygotes from homozygotes. Genotypes for these loci were not included in the mapping data.

\section{FIRST GENERATION OF AN INTRA-SPECIFIC MAP OF WILD LENTIL}

This study resulted in the generation of the first intra-specific gene-based map of wild lentil, established using a population of $\mathrm{F}_{2}$ individuals of a cross between two wild Le accessions, L01827a and IG 72815. The map consists of seven linkage groups, which likely represent the seven chromosomes of Le. Many of the early lentil maps were constructed using populations derived from inter-specific crosses between cultivated lentil and a wild parent from the secondary gene pool (Havey and Muehlbauer, 1989a,b; Tahir et al., 1993; Eujayl et al., 1998) to generate sufficient 
numbers of polymorphic markers. The recent development of improved marker technologies has enabled mapping with intraspecific crosses within the primary gene pool (Phan et al., 2006; Tullu et al., 2006, 2008; Fedoruk et al., 2013; Sharpe et al., 2013). To date, no maps of any of the wild lentil species have been published.

An important feature of existing lentil genetic maps is the clustering of markers in the middle, upper-middle, or lower-middle part of linkage groups indicative of reduced recombination in these regions (Hamwieh et al., 2005; Gupta et al., 2012). Similar clusters of markers were observed in the Le map in the current study, and in the recent SNP linkage map of cultivated lentil (Sharpe et al., 2013) containing more markers, both of which use gene-based markers. Such regions may span the location of the centromeres, which are often gene-poor and exhibit suppressed recombination.

The Le map from the current study consists of 377 gene-based markers of which 359 are TOGs that have been mapped in at least two other legume species. Apart from the model legumes with fully sequenced genomes, these markers have also been mapped in chickpea (Hiremath et al., 2012), common bean (Blair et al., 2013) and pigeon pea (Saxena et al., 2012), which should improve the ability to make cross-generic comparisons.

\section{COMPARATIVE MAPPING WITH CLOSELY RELATED LEGUMES SPECIES}

Through comparative mapping, predicting gene order and gene content across members of the same family is possible (Tanksley et al., 1992; Livingstone et al., 1999; Wilson et al., 1999). Lentil has been compared to Mt and pea using isozymes (Weeden et al., 1992) and large blocks of conserved synteny were demonstrated. Phan et al. (2006), Alo et al. (2011), and Gupta et al. (2012) use gene-based SSRs to compare Mt and Lc. Sharpe et al. (2013) confirm the extensive level of collinearity between $\mathrm{Mt}$ and Lc using gene-based SNP markers. The availability of single copy conserved sequences and markers for orthologous genes from heterologous legume species increases the reliability of comparative maps. Legume TOG markers have been used to study evolution across pigeon pea (Kassa et al., 2012), common bean (Blair et al., 2013) and other legumes (Choi et al., 2004a,b). The set of TOGs used in this study allows these comparisons to extend to wild lentil as well.

\section{CONSERVED SYNTENY BETWEEN L. ERVOIDES AND M. TRUNCATULA AND C. ARIETINUM}

Several research groups have compared the genome structure of Mt and various crop legumes (Choi et al., 2004a,b; Zhu et al., 2005). Lentil and Mt, its most closely related model legume species, diverged some 24 (Lavin et al., 2005) to 38 (Sharpe et al., 2013) million years ago. By using the TOG markers, a comparative analysis was carried out to demonstrate a high level of conserved macrosynteny between Le and Mt (Figure 3B). With the exception of Mt chromosome 6, extensive collinearity between the two species was evident. Mt chromosome 6 is unusual in that it has very few genes and appears to have originated as a fragment of a larger chromosome of the ancestral legume (Kalo et al., 2004; Cannon et al., 2006; Young et al., 2011). This Mt chromosome appears to have broken away from the ancestor of linkage group
2 in both Le (Figures 3B, 4A) and Lc (Sharpe et al., 2013) and chickpea (Additional file 3 ).

A large inversion in the middle of LeLG1 relative to Mt chromosome 1 is also found in Lc (Figure 4B). The only other notable exception to this collinearity is a translocation involving Mt chromosomes 4 and 8 relative to LeLG4 and LeLG7. Kamphuis et al. (2007) provide strong evidence of a reciprocal translocation in the sequenced Mt reference genotype A17 involving chromosomes 4 and 8 that is not present in other Mt accessions. This suggests the break in collinearity seen in Le relative to A17 is a specific consequence of chromosomal rearrangements in genotype A17, rather than that characteristic of species $\mathrm{Mt}$ or in the common ancestor of Medicago and Lens.

A set of the legume TOGs was identified and mapped in chickpea (Hiremath et al., 2012), allowing a comparison of common markers in all three genomes (Figure 3; Additional file 3). All 128 TOGs mapped in all three species and shows a high level of conservation of synteny among the species and can also be used as a resource to identify syntenic regions in other species. Clear blocks of shared synteny cover all linkage groups of the three species and key chromosomal rearrangements distinguish them. The identification of conserved regions relative to these fully sequenced legumes will aid more detailed study of legume genome evolution and the comparative analysis of the genic basis of traits. It is now also possible to use comparative information from these bettercharacterized species to accelerate candidate gene identification in lentil.

\section{ANCIENT TRANSLOCATION IN THE GENUS LENS}

New species arise when plants are reproductively isolated. Over time, chromosomal rearrangements preclude them from being inter-fertile when reintroduced to a common environment. Le and Lc diverged less than a million years ago (Sharpe et al., 2013) but have already undergone chromosomal rearrangements that make the production of fully fertile offspring difficult (Tadmor et al., 1987; Weeden et al., 1992).

The gene-based linkage map of Le was compared with a map of Lc (Fedoruk et al., 2013) using Mt as an intermediate, a necessary step because there were few polymorphic TOGs in Lc. Even fewer polymorphisms could be mapped in the Lc mapping population LR-18, resulting in few common markers between the two species. Because the Le map consisted of gene-based TOG and SNP markers, identifying orthologs in the fully sequenced Mt genome was possible. Comparative mapping showed a direct and simple relationship between Le linkage groups and Mt chromosomes. For Lc, however, the comparison appeared more complex but this is likely a result of the comparison being less robust due to the source of the SNP markers used in that map. Those SNPs were derived from $3^{\prime}$ end sequencing of transcripts (Sharpe et al., 2013) whose divergent nature makes comparisons to Mt difficult. In addition, the limited dataset available for Lc means some of the Homologs identified using reciprocal BLAST could have been paralogs, leading to less well-defined collinearity (Figure 4A). Despite this, it was still possible to identify gross level rearrangements that define the two species.

Previous studies in Lc and Le established the foundation of a single ancient translocation that separates the two species 
(Ladizinsky et al., 1985; Tadmor et al., 1987; Weeden et al., 1992). The reciprocal translocation observed on linkage groups 1 and 5 of Lc with respect to Mt (Sharpe et al., 2013; Figure 4B) is not evident in Le and likely represents this key difference between the two Lens species. The cotyledon color gene, $Y c$, maps to LcLG1 (Fedoruk et al., 2013) near to the breakpoint of this translocation. A similar observation was made by Weeden et al. (1992) based on mapping of interspecific hybrids between Lc and Le. They identify translocation break point using morphological $(Y c)$ and isozyme markers (Pgm-2, Aco-1). The first $5 \mathrm{cM}$ of LcLG1 have Homologs from Mt chromosome 5. There is a major gap of $16.7 \mathrm{cM}$ in this region in Lc, which likely reflects a loss of chromosomal segment or re-arrangement in the Lc genome. Similarly for LcLG5, a group of markers that mapped between $64.4 \mathrm{cM}$ and $100.2 \mathrm{cM}$ corresponds to Homologs from Mt chromosome 1. No such translocation was evident in Le relative to Mt (Figure 4B), suggesting this is unique to Lc.

The presence of a translocation in Lc that is not present in Le is corroborated by segregation of TOG loci in an interspecific RIL population, LR-59, derived from an Lc $\times$ Le cross. In this population, markers at the top of one LR-59 linkage group correspond to LeLG1 while the rest of the linkage group corresponds to markers mapping to LeLG5 (Figure 5). Many of the markers on this LR-59 linkage group were distorted toward the Lc parent alleles, suggesting preference for the Lc structure during meiotic events that occurred during the development of the inbred lines. Heterozygous genotypes that are atypical for RILs, were observed in the region surrounding the putative break point, suggesting incomplete pairing and recombination during meiosis as would happen in the case of mis-matched chromosomes. Segregation distortion and unequal crossing over as well as the presence of transpositional elements have been associated with chromosomal rearrangements in interspecific Lens hybrids (Galasso, 2003) and confirmed here for Le and Lc.

The translocation observed between Le and Mt involving Mt chromosomes 4 and 8 is absent in Lc based on the LR-18 map (Figure 4C). This suggests the presence of a second translocation distinguishing Le from Lc which had not been observed previously. Genotypic data from these regions in an additional Lc mapping population, however, indicated the presence of additional markers, monomorphic in LR-18, that fall in the region spanning the translocation in Mt and confirm that Lc and Le share the same structure with no evidence in our data of additional translocations. Genotypic data from the LR-59 interspecific population corresponding to these two linkage groups were not distorted toward one or other parental allele and were completely collinear with LeLG 4 and LeLG 7, further confirming the lack of translocation in this region within Lc relative to Le. The lack of a translocation further confirms the observations of Kamphuis et al. (2007) that A17 has a unique translocation and suggests that the common ancestor to both Mt and Lens share similar structure in these two chromosomes.

\section{SUMMARY}

With the exception of this one major translocation, the other five linkage groups appear to be collinear, suggesting interspecies hybrids could be used to effectively introgress genes from wild lentil into cultivated lentil unless they occur in or near the translocation breakpoint. Mapping of genes or traits of interest in LR-66 or other Le populations will allow for the development of markers that can be used to track introgression following crossing into cultivated lentil and facilitate selection for individuals carrying the Le alleles in the regions of interest. The construction of a genetic linkage map of the wild lentil L. ervoides and the identification of the chromosomal changes that differentiate it from cultivated lentil provide lentil geneticists a bridge to the genomic information and the genetic resources of better-characterized legumes. Knowledge of the colinearity among these legume species will allow us to leverage information from fully sequenced species for molecular marker-based breeding and discovery of genetic basis of traits in Lens species.

\section{AUTHOR CONTRIBUTIONS}

Kirstin E. Bett, Albert Vandenberg, and Douglas R. Cook were PIs on the projects that led to this manuscript. Douglas R. Cook, R. Varma Penmetsa, Noelia Carrasquilla-Garcia, and Andrew D. Farmer developed the legume COS markers. Andrew D. Farmer developed the bioinformatic pipelines. Albert Vandenberg selected the genotypes for sequencing and Sally L. Vail developed the LR-66 mapping population. Sally L. Vail, Noelia Carrasquilla-Garcia, and R. Varma Penmetsa carried out the initial sequencing of the four Lens genotypes. Kirstin $\mathrm{E}$. Bett, Andrew D. Farmer, and R. Varma Penmetsa designed the GoldenGate OPA. Kirstin E. Bett analyzed the GoldenGate data and did the genome mapping with Neha Gujaria-Verma. Andrew D. Farmer, Kirstin E. Bett, and Neha Gujaria-Verma did the crossspecies comparisons. Neha Gujaria-Verma and Kirstin E. Bett wrote the first draft of the manuscript.

\section{FUNDING}

The authors acknowledge funding from the Saskatchewan Pulse Growers (BRE0820) to Kirstin E. Bett, Albert Vandenberg, and Sally L. Vail and from the US National Science Foundation DBI 0605251 to Douglas R. Cook.

\section{ACKNOWLEDGMENTS}

Technical support: Lacey-Anne Sanderson, Carmen Breitkreutz, Rui Song, Rob Stonehouse, P. Vijayan, Jimmy Woodward, and Larissa Ramsay.

\section{SUPPLEMENTARY MATERIAL}

The Supplementary Material for this article can be found online at: http://www.frontiersin.org/journal/10.3389/fpls.2014.00676/ abstract

All contigs and identified SNPs are available through the KnowPulse webportal at http://knowpulse2.usask.ca/portal/ node $/ 53$ and in Additional file $1 \mathrm{~B}$.

\section{REFERENCES}

Ahmadian, A., Gharizadeh, B., Gustafsson, A. C., Sterk, F., Nyrén, P., Uhlén, M., et al. (2000). Single-nucleotide polymorphism analysis by pyrosequencing. Anal. Biochem. 280, 103-110. doi: 10.1006/abio.2000.4493

Alo, F., Furman, B. J., Akhunov, E., Dvorak, J., and Gepts, P. (2011). Leveraging genomic resources of model species for the assessment of diversity and phylogeny in wild and domesticated lentil. J. Hered. 102, 315-329. doi: 10.1093/jhered/esr015 
Andersen, J. R., and Lübberstedt, T. (2003). Functional markers in plants. Trends Plant Sci. 8, 554-560. doi: 10.1016/j.tplants.2003.09.010

Arumuganathan, K., and Earle, E. D. (1991). Nuclear DNA content of some important plant species. Mol. Biol. Report. 9, 208-221. doi: 10.1007/BF02672069

Bayaa, B., Erskine, W., and Hamdi, A. (1994). Response of wild lentil to Ascochyta fabae f.sp. lentis from Syria. Genet. Resour. Crop Evol. 41, 61-65. doi: 10.1007/BF00053049

Bayer, M., Milne, I., Stephen, G., Shaw, P., Cardle, L., Wright, F., et al. (2011). Comparative visualization of genetic and physical maps with strudel. Bioinformatics 27, 1307-1308. doi: 10.1093/bioinformatics/btr111

Blair, M. W., Cortés, A. J., Penmetsa, R. V., Farmer, A., Carrasquilla-Garcia, N., and Cook, D. R. (2013). A high-throughput SNP marker system for parental polymorphism screening, and diversity analysis in common bean (Phaseolus vulgaris L.). Theor. Appl. Genet. 126, 535-548. doi: 10.1007/s00122-012-1999-z

Cannon, S. B., May, G. D., and Jackson, S. A. (2009). Three sequenced legume genomes and many crop species: rich opportunities for translational genomics. Plant Physiol. 151, 970-977. doi: 10.1104/pp.109.144659

Cannon, S. B., Sterck, L., Rombauts,. S., Sato, S., Cheung, F., Gouzy, J., et al. (2006). Legume genome evolution viewed through the Medicago truncatula and Lotus japonicus genomes. Proc. Natl. Acad. Sci. U.S.A. 103, 14959-14964. doi: 10.1073/pnas.0603228103

Chen, W., Mingus, J., Mammadov, J., Backlund, J. E., Greene, T., Thompson, S., et al. (2010). "KASPar: a simple and cost effective system for SNP genotyping," in Plant and Animal Genomes (PAG) XVII Conference (San Diego, CA), 9-13.

Choi, H. K., Kim, D., Uhm, T., Limpens, E., Lim, H., Mun, J. H., et al. (2004a). A sequence-based genetic map of Medicago truncatula and comparison of marker collinearity with M. sativa. Genetics 166, 1463-1502. doi: 10.1534/genetics.166.3.1463

Choi, H. K., Luckow, M. A., Doyle, J., and Cook, D. R. (2006). Development of nuclear gene-derived molecular markers linked to legume genetic maps. Mol. Genet. Genomics 276, 56-70. doi: 10.1007/s00438-006-0118-8

Choi, H. K., Mun, J. H., Kim, D. J., Zhu, H., Baek, J. M., Mudge, J., et al. (2004b). Estimating genome conservation between crop and model legume species. Proc. Natl. Acad. Sci. U.S.A. 101, 15289-15294. doi: 10.1073/pnas.04022 51101

Cornille, A., Gladieux, P., Smulders, M. J. M., Roldán-Ruiz, I., Laurens, F., Le Cam, B., et al. (2012). New insight into the history of domesticated apple: secondary contribution of the European wild apple to the genome of cultivated varieties. PLoS Genet. 8:e1002703. doi: 10.1371/journal.pgen.1002703

Deulvot, C., Charrel, H., Marty, A., Jacquin, F., Donnadieu, C., Lejeune-He'naut, I., et al. (2010). Highly-multiplexed SNP genotyping for genetic mapping and germplasm diversity studies in pea. BMC Genomics 11:468. doi: 10.1186/14712164-11-468

Doyle, J. J., and Doyle, J. L. (1990). Isolation of plant DNA from fresh tissue. Focus $12,13-15$.

Erskine, W. (1996). Lessons for breeders from land races of lentil. Euphytica 93, 107-112. doi: 10.1023/A:1002939704321

Eujayl, I., Baum, M., Powell, W., Erskine, W., and Pehu, E. (1998). A genetic linkage map of lentil (Lens sp.) based on RAPD and AFLP markers using recombinant inbred lines. Theor. Appl. Genet. 97, 83-89. doi: 10.1007/s0012 20050869

Fedoruk, M. J., Vandenberg, A., and Bett, K. E. (2013). QTL analysis of seed quality characteristics in lentil (Lens culinaris ssp. culinaris Medik.) using SNP markers. Plant Gen. 6:3. doi:10.3835/plantgenome2013.05.0012

Fiala, J. V., Tullu, A., Banniza, S., Séguin-Swartz, G., and Vandenberg, A. (2009). Interspecies transfer of resistance to anthracnose in lentil (Lens culinaris Medic.). Crop Sci. 49, 825-830. doi: 10.2135/cropsci2008.05.0260

Fulton, T., Van der Hoeven, R., Eannetta, N., and Tanksley, S. (2002). Identification, analysis, and utilization of conserved ortholog set markers for comparative genomics in higher plants. Plant Cell 14, 1457-1467. doi: 10.1105/tpc. 010479

Galasso, I. (2003). Distribution of highly repeated DNA sequences in species of the genus Lens Miller. Genome 46, 1118-1124. doi: 10.1139/g03-077

Gaur, R., Azam, S., Jeena, G., Khan, A. W., Choudhary, S., Jain, M., et al. (2012). High-throughput SNP discovery and genotyping for constructing a saturated linkage map of chickpea (Cicer arietinum L.). DNA Res. 19, 357-373. doi: 10.1093/dnares/dss018

Gujaria, N., Kumar, A., Dauthal, P., Dubey, A., Hiremath, P., Bhanu Prakash, A., et al. (2011). Development and use of genic molecular markers
(GMMs) for construction of a transcript map of chickpea (Cicer arietinum L.). Theor. Appl. Genet. 122, 1577-1589. doi: 10.1007/s00122-0111556-1

Gupta, M., Verma, B., Kumar, N., Chahota, R. K., Rathour, R., Sharma, S. K., et al. (2012). Construction of intersubspecific molecular genetic map of lentil based on ISSR, RAPD and SSR markers. J. Genet. 91, 279-287. doi: 10.1007/s12041012-0180-4

Gupta, P. K., and Varshney, R. K. (2000). The development and use of microsatellite markers for genetic analysis and plant breeding with emphasis on bread wheat. Euphytica 113, 163-185. doi: 10.1023/A:1003910819967

Hamwieh, A., Udupa, S. M., Choumane, W., Sarker, A., Dreyer, F., Jung, C., et al. (2005). A genetic linkage map of Lens spp. based on microsatellite and AFLP markers and the localization of Fusarium vascular wilt resistance. Theor. Appl. Genet. 110, 669-677. doi: 10.1007/s00122-004-1892-5

Havey, M. J., and Muehlbauer, F. J. (1989a). Linkages between restriction fragment length, isozyme, and morphological markers in lentil. Theor. Appl. Genet. 77, 395-401. doi: 10.1007/BF00305835

Havey, M. J., and Muehlbauer, F. J. (1989b). Variability for restriction fragment lengths and phylogenies in lentil. Theor. Appl. Genet. 77, 839-843. doi 10.1007/BF00268336

Hiremath, P. J., Kumar, A., Penmetsa, R. V., Farmer, A., Schlueter, J. A., Chamarthi, S. K., et al. (2012). Large-scale development of cost-effective SNP marker assays for diversity assessment and genetic mapping in chickpea and comparative mapping in legumes. Plant Biotechnol. J. 10, 716-732. doi: 10.1111/j.14677652.2012.00710.x

Huang, X., and Madan, A. (1999). Cap3: a DNA sequence assembly program. Genome Res. 9, 868-877. doi: 10.1101/gr.9.9.868

Hufford, M. B., Lubinksy, P., Pyhäjärvi, T., Devengenzo, M. T., Ellstrand, N. C., and Ross-Ibarra, J. (2013). The genomic signature of crop-wild introgression in maize. PLoS Genet. 9:e1003477. doi: 10.1371/journal.pgen.1003477

Hyten, D. L., Choi, I. Y., Song, Q., Specht, J. E., Carter, T. E. Jr., Shoemaker, R. C., et al. (2010). A high density integrated genetic linkage map of soybean and the development of a 1536 universal soy linkage panel for quantitative trait locus mapping. Crop Sci. 50, 960-968. doi: 10.2135/cropsci2009. 06.0360

Hyten, D. L., Song, Q., Choi, I. Y., Yoon, M. S., Specht, J. E., Matukumalli, L. K., et al. (2008). High throughput genotyping with the GoldenGate assay in the complex genome of soybean. Theor. Appl. Genet. 116, 945-952. doi: 10.1007/s00122-008-0726-2

Ishikawa, S., Komura, D., Tsuji, S., Nishimura, K., Yakamoto, S., Panda, B., et al. (2005). Allelic dosage analysis with genotyping microarrays. Biochem. Biophys. Res. Commun. 333, 1309-1314. doi: 10.1016/j.bbrc.2005.06.040

Kalo, P., Seres, A., Taylor, S. A., Jakab, J., Kevei, Z., Kereszt, et al. (2004) Comparative mapping between Medicago sativa and Pisum sativum. Mol. Genet. Genomics 272, 235-246. doi: 10.1007/s00438-004-1055-Z

Kamphuis, L. G., Williams, A. H., D’Souza, N. K., Pfaff, T., Ellwood, S. R., Groves, E. J., et al. (2007). The Medicago truncatula reference accession A17 has an aberrant chromosomal configuration. New Phytol. 174, 299-303. doi: 10.1111/j.1469-8137.2007.02039.x

Kassa, M. T., Penmetsa, R. V., Carrasquilla-Garcia, N., Sarma, B. K., Datta, S., Upadhyaya, H. D., et al. (2012). Genetic patterns of domestication in pigeonpea (Cajanus cajan (L.) Millsp.) and wild Cajanus relatives. PLoS ONE 7:e39563. doi: 10.1371/journal.pone. 0039563

Kaur, S., Cogan, N. O., Pembleton, L. W., Shinozuka, M., Savin, K. W., Materne, M., et al. (2011). Transcriptome sequencing of lentil based on second-generation technology permits large-scale unigene assembly and SSR marker discovery. BMC Genomics 12:265. doi: 10.1186/1471-2164-12-265

Kaur, S., Cogan, N. O., Stephens, A., Noy, D., Butsch, M., Forster, J. W., et al. (2013). EST-SNP discovery and dense genetic mapping in lentil enable candidate gene selection for boron tolerance. Theor. Appl. Genet. 127, 703-713. doi: 10.1007/s00122-013-2252-0

Krzywinski, M., Schein, J. E., Birol, I., Connors, J., Gascoyne, R., Horsman, D., et al. (2009). Circos: an information aesthetic for comparative genomics. Genome Res. 19, 1639-1645. doi: 10.1101/gr.092759.109

Kurtz, S., Phillippy, A., Delcher, A. L., Smoot, M., Shumway, M., Antonescu, C., et al. (2004). Versatile and open software for comparing large genomes. Genome Biol. 5:R12. doi: 10.1186/gb-2004-5-2-r12

Ladizinsky, G., Braun, D., Goshen, D., and Muehlbauer, F. J. (1984). The biological species in the genus Lens. Bot Gaz. 145, 253-261. doi: 10.1086/337454 
Ladizinsky, G., Cohen, D., and Muehlbauer, F. J. (1985). Hybridization in the genus Lens by means of embryo culture. Theor. Appl. Genet. 70, 97-101. doi: 10.1007/BF00264489

Lavin, M., Herendeen, P. S., and Wojciechowski, M. F. (2005). Evolutionary rates analysis of Leguminosae implicates a rapid diversification of lineages during the tertiary. Syst Biol. 54, 575-594. doi: 10.1080/10635150590947131

Livingstone, K. D., Lackney, V. K., Blauth, J. R., van Wijk, R., and Jahn, M. K. (1999). Genome mapping in Capsicum and the evolution of genome structure in the Solanaceae. Genetics 152, 1183-1202.

Mardis, E. R. (2008). Next-generation DNA sequencing methods. Annu. Rev. Genomics Hum. Genet. 9, 387-402. doi: 10.1146/annurev.genom.9.081307. 164359

Metzker, M. L. (2009). Sequencing technologies - the next generation. Nat. Rev. Genet. 11, 31-46. doi: 10.1038/nrg2626

Muchero, W., Diop, N. N., Bhat, P. R., Fenton, R. D., Wanamaker, S., Pottor, M., et al. (2009). A consensus genetic map of cowpea [Vigna unguiculata Walp.] and synteny based on EST-derived SNPs. Proc. Natl. Acad. Sci. U.S.A. 106, 18159-18164. doi: 10.1073/pnas.0905886106

Oliphant, A., Barker, D. L., Stuelpnagel, J. R., and Chee, M. S. (2002). BeadArray technology: enabling an accurate, cost-effective approach to high throughput genotyping. Biotechniques (Suppl. 5), 32, S56-S61.

Phan, H. T. T., Elwood, S. R., Hane, J. K., Ford, R., Materne, M., and Oliver, R. P. (2006). Extensive macrosynteny between Medicago truncatula and Lens culinaris ssp. culinaris. Theor. Appl. Genet. 114, 549-558. doi: 10.1007/s00122-006-0455-3

Podder, R., Banniza, S., and Vandenberg, A. (2013). Screening of wild and cultivated lentil germplasm for resistance to stemphylium blight. Plant Genet. Resour. 11, 26-35. doi: 10.1017/S1479262112000329

Ronaghi, M., Uhlen, M., and Nyren, P. (1998). A sequencing method based on realtime pyrophosphate. Science 281:363. doi: 10.1126/science.281.5375.363

Rostoks, N., Ramsay, L., MacKenzie, K., Cardle, L., Bhat, P. R., Roose, M. L., et al. (2006). Recent history of artificial outcrossing facilitates whole-genome association mapping in elite inbred crop varietes. Proc. Natl. Acad. Sci. U.S.A. 103, 18656-18661. doi: 10.1073/pnas.0606133103

Sapolsky, R. J., Hsie, L., Berno, A., Ghandour, G., Mittmann, M., and Fan, J. B. (1999). High-throughput polymorphism screening and genotyping with high density oligonucleotide arrays. Genet. Anal. Biomol. Eng. 14, 187-192. doi: 10.1016/S1050-3862(98)00026-6

Sawkins, M. C., Farmer, A. D., Hoisington, D., Sullivan, J., Tolopko, A., Jiang, Z., et al. (2004). Comparative map and trait viewer (CMTV): an integrated bioinformatic tool to construct consensus maps and compare QTL and functional genomics data across genomes and experiments. Plant Mol. Biol. 56, 465-480. doi: 10.1007/s11103-004-4950-0

Saxena, R. K., Penmetsa, R. V., Upadhyaya, H. D., Kumar, A., CarrasquillaGarcia, N., Schlueter, J. A., et al. (2012). Large-scale development of costeffective single-nucleotide polymorphism marker assays for genetic mapping in pigeonpea and comparative mapping in legumes. DNA Res. 19, 449-461. doi: 10.1093/dnares/dss025

Sharpe, A. G., Ramsay, L., Sanderson, L. A., Fedoruk, M. J., Clarke, W. E., Li, R., et al. (2013). Ancient orphan crop joins modern era: gene-based SNP discovery and mapping in lentil. BMC Genomics 14:192. doi: 10.1186/1471-2164-14-192

Slinkard, A. E. (1981). Eston lentil. Can. J. Plant Sci. 61, 733-734. doi: 10.4141/cjps81-104

Tadmor, Y., Zamir, D., and Ladizinsky, G. (1987). Genetic mapping of an ancient translocation in the genus Lens. Theor. Appl. Genet. 73, 883-892. doi: 10.1007/BF00289394

Tahir, M., Simon, C. J., and Muehlbauer, F. J. (1993). Gene map of lentil: a review. Lens Newsl. 20, 3-10.

Tanksley, S. D., Ganal, M. W., Prince, J. P., de Vicente, M. C., Bonierbale, M. W., Broun, P., et al. (1992). High density molecular linkage maps of the tomato and potato genomes. Genetics 132, 1141-1160.

Tullu, A., Buchwaldt, L., Lulsdorf, M., Banniza, S., Barlow, B., Slinkard, A. E., et al. (2006). Sources of resistance to anthracnose (Colletotrichum truncatum) in wild Lens species. Genet. Resour. Crop Evol. 53, 111-119. doi: 10.1007/s10722-0041586-5

Tullu, A., Tar'an, B., Warkentin, T., and Vandenberg, A. (2008). Construction of an intraspecific linkage map and QTL analysis for earliness and plant height in lentil. Crop Sci. 48, 2254-2264. doi: 10.2135/cropsci2007.11.0628

Vail, S. L., Tullu, A., and Vandenberg, A. (2011). Field evaluation of resistance to Colletotrichum truncatum in Lens culinaris, Lens ervoides, and Lens ervoides x Lens culinaris derivatives. Field Crops Res. 126, 145-151. doi: 10.1016/j.fcr.2011.10.002

Vandenberg, A., Banniza, S., Warkentin, T. D., Ife, S., Barlow, B., McHale, S., et al. (2006). CDC Redberry lentil. Can. J. Plant Sci. 86, 497-498. doi: 10.4141/ P05-071

Van Ooijen, J. W. (2006). JoinMap ${ }^{\circledR}$ 4, Software for the Calculation of Genetic Linkage Maps in Experimental Populations. Wageningen: Kyazma BV.

Varshney, R. K. (2010). "Gene-based marker systems in plants: high throughput approaches for discovery and genotyping," in Molecular Techniques in Crop Improvement, eds. S. M. Jain and D. S. Brar (Dodrecht; Heidelberg; London; New York: Springer), 119-142.

Varshney, R. K., Hoisington, D. A., Nayak, S. N., and Graner, A. (2009). "Molecular plant breeding: methodology and achievements," in Plant Genomics: Methods and Protocols, eds D. Somers, P. Langridge, and P. J. Gustafson (Totowa, NJ: The Humana Press), 283-304.

Voorrips, R. E. (2002). MapChart: software for the graphical presentation of linkage maps and QTLs. J. Hered. 93, 77-78. doi: 10.1093/jhered/93.1.77

Weeden, N. F., Muehlbauer, F. J., and Ladizinsky, G. (1992). Extensive conservation of linkage relationships between pea and lentil genetic maps. J Hered. 83, 123-129.

Wilson, W. A., Harrington, S. E., Woodman, W. L., Lee, M., Sorrells, M. E., and McCouch, S. R. (1999). Inferences on the genome structure of progenitor maize through comparative analysis of rice, maize and the domesticated panicoids. Genetics 153, 453-473.

Wu, T. D., and Watanabe, C. (2005). GMAP: a genomic mapping and alignment program for mRNA and EST sequences. Bioinformatics 21, 1859-1875. doi: 10.1093/bioinformatics/bti310

Yan, J. B., Yang, X. H., Shah, T., Sanchez-Villeda, H., Li, J. S., Warburton, M., et al. (2010). High-throughput SNP genotyping with the GoldenGate assay in maize. Mol. Breed. 25, 441-451. doi: 10.1007/s11032-0099343-2

Young, N., Debellé, F., Oldroyd, G. E. D., Geurts, R., Cannon, S. B., Udvardi, M. K., et al. (2011). The Medicago genome provides insight into the evolution of rhizobial symbioses. Nature 480, 520-524. doi: 10.1038/ nature 10625

Zhao, K., Wright, M., Kimball, J., Eizenga, G., McClung, A., Kovach, M., et al. (2010). Genomic diversity and introgression in O. sativa reveal the impact of domestication and breeding on the rice genome. PLOS ONE 5:e10780. doi: 10.1371/journal.pone.0010780

Zhu, H., Choi, H. K., Cook, D. R., and Shoemaker, R. C. (2005). Bridging model and crop legumes through comparative genomics. Plant Physiol. 137, 1189-1196. doi: 10.1104/pp.104.058891

Conflict of Interest Statement: The Associate Editor Paul Gepts declares that, despite being affiliated to the same institution as authors Sally L. Vail, Noelia Carrasquilla-Garcia, R. Varma Penmetsa and Douglas R. Cook, the review process was handled objectively and no conflict of interest exists. The authors declare that the experiments of this study comply with the current laws. We confirm to have the authority to publish this work and that the manuscript has not been published before and is not under consideration for publication elsewhere. The authors declare that the research was conducted in the absence of any commercial or financial relationships that could be construed as a potential conflict of interest.

Received: 01 October 2014; paper pending published: 08 November 2014; accepted: 13 November 2014; published online: 05 December 2014.

Citation: Gujaria-Verma N, Vail SL, Carrasquilla-Garcia N, Penmetsa RV, Cook DR, Farmer AD, Vandenberg A and Bett KE (2014) Genetic mapping of legume orthologs reveals high conservation of synteny between lentil species and the sequenced genomes of Medicago and chickpea. Front. Plant Sci. 5:676. doi: 10.3389/fpls.2014.00676

This article was submitted to Plant Genetics and Genomics, a section of the journal Frontiers in Plant Science.

Copyright (C) 2014 Gujaria-Verma, Vail, Carrasquilla-Garcia, Penmetsa, Cook, Farmer, Vandenberg and Bett. This is an open-access article distributed under the terms of the Creative Commons Attribution License (CC BY). The use, distribution or reproduction in other forums is permitted, provided the original author(s) or licensor are credited and that the original publication in this journal is cited, in accordance with accepted academic practice. No use, distribution or reproduction is permitted which does not comply with these terms. 